\title{
Número 4 Mayo 2012: La ciudad de abajo arriba. Aportaciones para la práctica y la teoría del urbanismo participativo
}

\section{Coordinadores}

Carlos Verdaguer Viana-Cárdenas, Isabela Velázquez Valoria

\section{Presentación del número 4}

Carlos Verdaguer Viana-Cárdenas, GEA 21, Universidad Politécnica de Madrid, Arquitecto

Isabela Velázquez Valoria, GEA 21, Madrid, Arquitecta

\section{Urbanismo y participación: una lenta convergencia}

La necesidad de recuperar la ciudad como construcción colectiva y de ampliar los escenarios de apropiación de lo urbano por parte de la ciudadanía aparece cada vez más como una herramienta fundamental para hacer frente desde la óptica de la sostenibilidad al embate de una crisis múltiple que engloba lo económico, lo social y lo ambiental y que se encarna de forma especialmente virulenta en la realidad urbanoterritorial. Hoy más que nunca vemos en nuestras ciudades y campos los efectos desastrosos de todos aquellos procesos de transformación en los que la toma de decisiones se ha producido a gran distancia de quienes ahora los están sufriendo, y siguiendo lógicas muy ajenas a las necesidades y deseos de los ciudadanos.

Sin embargo, a pesar de que esta constatación ha acabado situándose cada vez más en primer plano en las reflexiones y prácticas más avanzadas en el campo del urbanismo, y de que la palabra participación se ofrece cada vez de forma más ubicua en muchos documentos y políticas institucionales, lo cierto es que nuestro país aún está muy lejos de haber salvado, en lo que respecta a la realidad cotidiana del urbanismo participativo, la gran distancia que lo separa de otros países europeos, en algunos de los cuales las primeras experiencias de calado se remontan a hace casi treinta años. Son múltiples las razones para este retraso, y muchas tienen que ver con el déficit en cultura democrática que ha sufrido la sociedad española durante varias generaciones y que ha dejado una profunda huella en nuestra forma de entender lo público.

Por otra parte, aunque puntuales y en general desconectadas, son ya numerosas las experiencias de urbanismo participativo a todas las escalas que se han producido en las últimas décadas en nuestro país, convirtiéndose algunas de ellas en incuestionables referencias; y es preciso señalar también que, en lo que respecta a la apuesta social e institucional por un urbanismo de los ciudadanos, las diferencias entre unas y otras realidades autonómicas empiezan a ser verdaderamente llamativas. Todo ello se traduce en un panorama desigual y heterogéneo que urge cartografiar para seguir avanzando.

\section{Temas clave para una nueva cultura del territorio}

Dentro de este panorama heterogéneo, atravesado fructíferamente por muchas líneas de reflexión desde las más diversas disciplinas, la participación aparece en ocasiones como un fin en sí misma, como una suerte de panacea o receta mágica abstracta que, al estar desprovista de objeto y objetivo, acaba también privada de contenido. La ventaja que aporta la reflexión desde la doble óptica de lo urbano y del paradigma ecológico es que puede contribuir a anclar de forma literal dichas reflexiones en la realidad física del terreno, del espacio construido y de los recursos, ayudando a hacer palpable la íntima ligazón que existe entre los procesos de tomas de decisiones, los flujos de materia y energía y los impactos sociales y 
económicos que entraña toda transformación de lo urbano y territorial.

Por ello, lo que se intenta en este número monográfico de Hábitat y Sociedad dedicado al urbanismo participativo es vincular estrechamente la reflexión sobre la forma en que se toman y se deben tomar las decisiones que competen a lo urbano y aquellos temas de sostenibilidad urbana que el actual panorama de crisis global ha contribuido a revelar como claves: la regeneración urbana integral como estrategia global de reciclaje y reutilización del tejido construido; el control del crecimiento urbano a partir de la redefinición de las señas de identidad y las relaciones entre el campo y la ciudad, entre el espacio urbano, el espacio agrícola, la naturaleza y el paisaje; la adecuación de las tipologías de vivienda a la riqueza y diversidad de modelos y perfiles sociales; la vitalización del espacio público como escenario privilegiado de la vida ciudadana, amenazado por los modelos cada vez más autistas de urbanización y movilidad y la incorporación de la lógica metabólica de flujos de energía, materia y residuos a la ordenación urbana.

Todos y cada uno de estos temas clave requieren de nuevas formas de entender la planificación y, una vez comprobados los desastrosos resultados de los modelos habituales basados en la transmisión de arriba abajo de las cadenas de decisiones, la participación ciudadana aparece en todos los casos de forma transversal como un factor y un instrumento imprescindible para la necesaria reconversión de nuestras teorías y prácticas urbanas.

Dentro del espacio necesariamente limitado de esta revista, no podemos aspirar sino a ofrecer una pequeña muestra del panorama heterogéneo que establece este marco conceptual, pero confiamos en que estas aportaciones contribuyan al menos a avanzar en el trazado de la cartografía del urbanismo participativo a la que antes hacíamos mención.

De acuerdo con este marco y estas limitaciones, la cosecha de artículos que se presenta en este número monográfico de Habitat y Sociedad se articula en términos generales en torno a dos ejes: el primero centrado en las experiencias emergentes a cargo principalmente de jóvenes profesionales y dentro de tres de los temas clave identificados, a saber, la regeneración urbana, la vivienda flexible y la agricultura urbana; y un segundo eje que pretende dar cuenta de los resultados obtenidos en aquellos territorios pioneros, como es el caso de Cataluña, donde la participación ha pasado a formar parte desde fecha relativamente reciente de la batería de herramientas normativas y legislativas asociadas al planeamiento urbanístico.

\section{Experiencias emergentes}

El primero de estos dos ejes ofrece una pequeña muestra del quehacer de ese conjunto cada vez más numeroso de equipos jóvenes que, haciendo virtud de la situación de estancamiento debida al post-tsunami inmobiliario en la que está sumido el sector, han encontrado el tiempo y la dedicación necesaria para experimentar y llevar a cabo experiencias de abajo a arriba en relación con los temas clave que centran este número. Iniciativas que, lamentablemente, a menudo cuentan con más ilusión y capacidad de trabajo personal y voluntario por parte de estos jóvenes profesionales que presupuestos de apoyo por parte de las instituciones y agencias públicas responsables del urbanismo. Si bien, en algunos casos, ha sido el apoyo de las instituciones académicas a través de investigaciones, tesis o seminarios de formación el que recurriendo a la filosofía del 'aprender haciendo' ha permitido mitigar el carácter exclusivamente voluntario de estos equipos comprometidos ética y personalmente con la participación. A favor de su visibilidad el dominio de las nuevas técnicas de difusión en la Web y en las redes sociales que poseen todos estos equipos y que les ha permitido otorgar una visibilidad en gran medida inédita a muchas de sus experiencias.

Es el caso del trabajo de Paisaje Transversal, que narra su interesante experiencia en el barrio madrileño de Virgen de Begoña, dando pie a una auto-reflexión sobre su inminente futuro como profesionales en el campo de la arquitectura y el urbanismo. Valga su relato como muestra representativa del trabajo de otras muchas asociaciones que actúan en este mismo ámbito en muchas ciudades españolas y cuyas iniciativas 
podrían haber estado igualmente presentes en esta recopilación.

Como sustitutivo de su ausencia aquí, es recomendable el acceso a sus espacios virtuales sumamente interesantes y sugerentes: así, merece la pena la revisión del trabajo también consolidado en años de trayectoria coherente de Otro Habitat en Madrid, coautores de la revista Márgenes; las intervenciones de Hiri Kolektiboak en Euskadi o de Col.lectiu Punt 6 en Cataluña, que poseen el valor añadido de su compromiso por la incorporación de la visión de género como elemento clave de la participación; el trabajo cada vez más interesante de Basurama, con iniciativas como e Autobarrios; la trayectoria de Zuloark, implicados en la generación de alternativas a la plaza de la Cebada en Madrid, o la comprometida mediación de Aldea Social junto a Todo por la Praxis en la complicada búsqueda de una salida al conflicto de la Cañada Real; o, en fin, el admirable proceso de recuperación de solares urbanos que ha impulsado con enorme efectividad en Zaragoza Patricia Di Monte a través de las intervenciones de 'Esto no es un Solar'.por retomar a los más reconocidos. Para completar este mosaico de experiencias y reflexiones sobre participación y ciudad construida habría que citar igualmente a profesionales de la arquitectura con una trayectoria ya consolidada. como Itziar González Virós, en el ámbito de la gestión político-ciudadana en el Ayuntamiento de Barcelona o a Vicente Díaz, con experiencias recientes de gran interés como la del barrio de la Isleta en las Palmas de Gran Canaria.

Una buena pista para acceder a este mosaico heterogéneo la ofrece la red de Arquitecturas Colectivas que está haciendo el esfuerzo de sumar energías arropando a muchos hilos de esta telaraña creativa y comprometida de grupos de toda condición, añada y tamaño, en un interesante trenzado de iniciativas y personas. No son los únicos: habría que traer a colación otros muchos grupos, cooperativas, asociaciones o profesionales por libre que nos ofrecen esta visión fresca y convincente de una nueva generación de arquitectos muy diferente de la dominante en las anteriores generaciones. En unos años, tendremos un completo balance de este nuevo modo de hacer, a menudo en colaboración estrecha con otros profesionales del trabajo social, de la educación, de la ecología o del arte.

En relación con la vivienda, la participación y la innovación, es imprescindible hacer mención también a una figura de referencia como es la de Santiago Cirugeda cuyas acciones y propuestas han contribuido a acelerar el cambio de mirada sobre la vivienda y el urbanismo. Como parte de este cambio y dentro también de esta batería de iniciativas innovadoras impulsadas por equipos jóvenes que articulan nuestro eje se incluye también en este número el caso de La Panadería, con su reflexión-investigación-participación sobre la vivienda en el entorno sevillano, incidiendo en conceptos clave para definir el nuevo ajuste entre vivienda y sociedad, en estos tiempos de desahucios de personas y viviendas vacantes expulsadas del mercado.

Otro de los temas claves relacionadas con la nueva cultura del territorio es la eclosión de ocupaciones sociales y ambientales del espacio público, para fines colectivos y transversales como es el impulso a la agricultura urbana. Como muestra de este ámbito, se presenta el artículo de la Red de Huertos Urbanos de Madrid, recién estrenada la valoración de esta iniciativa como Buena Práctica dentro de la edición de 2012 del Concurso Internacional de Buenas Prácticas de Naciones Unidas. El texto explica cómo en un plazo muy breve de tiempo han eclosionado en un ámbito tan árido como Madrid, una serie de espacios de gestión comunitaria que ofrecen oportunidades a todos aquellos ciudadanos interesados en múltiples objetivos relacionados todos con la calidad de vida urbana y el derecho a la ciudad, situando en primer plano un tema tan crucial como la alimentación. Esta eclosión autogestionaria es también representativa de las experiencias de otros muchos grupos en otros lugares de la geografía peninsular, anclados asimismo en asociaciones 
vecinales o en grupos de jóvenes activistas, como es el caso del huerto Miraflores o del huerto del Rey Moro en Sevilla.

Una vez cerrado este número monográfico, en relación con este eje de reflexión es inevitable experimentar una cierta percepción agridulce de haber abierto este espacio de expresión, por proximidad o disponibilidad y por los lógicos límites de espacio, sólo a una mínima expresión de la miríada de grupos y colectivos que están trabajando, cada uno con su mochila de experiencias e iniciativas, por cambiar la forma de intervención en la ciudad, y con los que sería interesante contar para sucesivos números de esta revista.....

\section{Avances en la institucionalización: el caso de Cataluña}

Por lo que se refiere al otro eje de reflexión, centrado en los avances en la institucionalización de la participación ciudadana, hay que constatar que se trata de un proceso mucho más lento y en el que las diferencias territoriales son palpables. Hemos elegido Cataluña como espacio en el que la participación está más normalizada dentro de nuestro entorno próximo, y en relación con este territorio tenemos la satisfacción de presentar una interesante reflexión a cargo del geógrafo David Mongil sobre la aplicación de procesos de participación en programas de tanto calado como son las actuaciones de mejora del espacio urbano y de los barrios, que ha impulsado la Llei de Barris, una ley pionera de cuya importancia e impacto no cabe ninguna duda.

Dentro de este mismo bloque de reflexión y sin salir del territorio catalán, presentamos también el relato por parte de Laura Giménez Gual de un interesante proceso, el Espai Laboratori, en el que a través de la mediación de la Diputación de Barcelona, se estableció un diálogo socrático entre equipos municipales (políticos y técnicos) y expertos en participación, para reflexionar sobre las conclusiones de los primeros proyectos de participación en planes urbanísticos fruto de la ley implantada que exige estos procesos. Consideramos que este proceso, en cuya organización tuvimos la fortuna de participar estrechamente los coordinadores de este número, reviste sobre todo el interés de haber puesto en primer plano un aspecto crucial al que el urbanismo estándar no suele prestar atención como es la retroalimentación y la evaluación continua de las experiencias en marcha para tratar de aprender de los aciertos y los errores, contribuyendo así a la calidad de la teoría y la práctica.

\section{Construyendo sobre la experiencia de todos}

Aunque el enfoque de esta revista se ha centrado deliberadamente en las experiencias emergentes por parte de equipos jóvenes y en los avances en la institucionalización de un ámbito territorial muy concreto, no queremos cerrar este número sin dejar constancia de que los avances en la incorporación de la participación ciudadana al proceso de construcción de la ciudad han sido posibles gracias, en primer lugar y naturalmente, a la propia presión desde abajo de los ciudadanos, pero también a la labor pionera de un conjunto de expertos de las más diversas disciplinas, constructores cuidadosos de este campo de conocimiento, por los que sentimos una profunda admiración y respecto y a cuyas reflexiones no hemos acudido en este caso porque consideramos que, afortunadamente, sus artículos, estudios, colaboraciones y publicaciones son cada vez de más fácil acceso a través de la red.

Y a este respecto, no queremos dejar de destacar la labor de investigación y reflexión que han desarrollado las universidades de Madrid, Barcelona y Sevilla, entre otras, donde los Master de Desarrollo Sostenible y Participación de la UAB, de Participación y desarrollo comunitario de Donostia-San Sebastián o Madrid y de 
Gestión Social del Habitat han desarrollado materias y documentos útiles para todos. O recordar la labor continuada de la Escuela de Políticas Sociales y Urbanas de Nou Barris y la investigación enmarcada en el IGOP con Oscar Rebollo y Subirats, así como el paulatino trabajo de Fernando Pindado en Barcelona, o la trayectoria de los equipos de Investigación-Acción Participativa que lidera desde hace décadas Tomás Rodríguez Villasante, o las aportaciones de personas y colectivos como Antígona, Etcs o Desenvolupament Comunitari, Ecotono; de académicos, de políticos y de activistas con los que hemos ido trabajando en sintonía, con poco tiempo para construir espacios de colaboración, pero poco a poco convergiendo en criterios, métodos, contenidos y, sobre todo, en coherencia y mejora continua de procesos que iniciamos con más ilusión y compromiso que profundidad y método. De bien nacidos es ser agradecidos y tenemos que agradecer la paulatina elaboración de contenidos y herramientas a aquellos que tienen la fortuna de investigar en estos temas.

Por nuestra parte, hemos de señalar que, debido en parte al retraso al que hacíamos mención al principio en lo que respecta a la convergencia entre urbanismo y participación, ha sido especialmente a través de nuestra colaboración en proyectos europeos, donde hemos encontrado las mejores oportunidades para reflexionar sobre las herramientas, conceptos y procesos de participación que utilizamos, siempre que se nos brinda la ocasión, en nuestro trabajo en el campo del urbanismo. Seguir mejorando la ciudad y aprendiendo todos de todos es el objeto de esta línea de trabajo que consideramos imprescindible en este momento de cambio ineludible en las formas de hacer ciudad e intervenir en el territorio.

http://dx.doi.org/10.12795/HabitatySociedad.2012.i4.01 